\title{
Tumor odontogénico queratoquístico. Hallazgo radiográfico y resolución quirúrgica de un caso clínico
}

\section{Keratocystic odontogenic tumor: Radiographic findings and surgical management of a clinical case}

\author{
Vázquez Diego J*, Gandini Pablo C**, Ramírez María J***, Ibero José N***, \\ Carbajal Eduardo $\mathrm{E}^{* * * * *}$
}

\section{RESUMEN}

Se presenta un caso clínico de queratoquiste odontogénico. El paciente de 26 años de edad es de sexo femenino. Se utilizan las técnicas radiológicas intrabucales y extrabucales para localizar y diagnosticar presuntivamente dicha patología. Posteriormente se realiza la biopsia que corrobora el diagnóstico presuntivo y se realizó intervención quirúrgica en la que se lleva a cabo la enucleación del tumor. Se realizaron los controles periódicos a corto, mediano y largo plazo en el que no se constató ninguna recidiva de la lesión. Basado en lo expuesto se analiza al tumor odontogénico queratoquístico según ubicación, sexo, edad y maxilar; habiendo realizado una revisión de la literatura.

Palabras clave: Tumor odontogénico queratoquístico, técnicas radiológicas, diagnóstico, tratamiento quirúrgico, caso clínico.

\section{SUMMARY}

We present a clinical case of odontogenic keratocyst in a 26 -year-old female patient. Both extraroal and intraoral radiographic techniques were used with the purpose of locating and attempt a presumptive diagnosis of this pathology. A biopsy was performed, confirming our earlier diagnostic presumption and then, surgical intervention was carried out, with the complete enucleation of the tumor. Short-, mid- and long-term controls were also performed, not showing any signs of a possible recurrence of the lesion. Based on our findings, we study the keratocystic odontogenic tumor according to location, gender, age and jaw affected, after reviewing the current literature on this issue.

Key words: Keratocystic odontogenic tumor, radiographic techniques, diagnosis, surgical treatment, clinical case.

Fecha de recepción: 2 de septiembre de 2010.

Aceptado para publicación: 15 de septiembre de 2010.

Vázquez Diego J, Gandini Pablo C, Ramírez María J, Ibero José N, Carbajal Eduardo E. Tumor odontogénico queratoquístico. Hallazgo radiográfico y resolución quirúrgica de un caso clínico. Av. Odontoestomatol 2012; 28 (5): 249-254.

\footnotetext{
* Jefe de Trabajos Prácticos. Cátedra de Radiología. Facultad de Odontología. Universidad de Buenos Aires. Especialista en Diagnóstico por Imágenes Buco-maxilo-facial

** Ayudante de Primera. Cátedra de Cirugía B. M. F. III. Facultad de Odontología. Universidad de Buenos Aires. Especialista en Cirugía Buco-maxilo-facial.

*** Ayudante de Primera. Cátedra de Radiología. Facultad de Odontología. Universidad de Buenos Aires.

**** Profesor Adjunto. Cátedra de Radiología. Facultad de Odontología. Universidad de Buenos Aires. Especialista en Diagnóstico por Imágenes Buco-maxilo-facial.
} 


\section{INTRODUCCIÓN}

El Tumor odontogénico queratoquístico es una neoplasia odontogénica benigna, asintomática, de larga evolución, única o múltiples que presentan un alto grado de recidiva. Esta lesión fue descrita por primera vez por Philipsen en 1956 con el nombre de queratoquiste. En el año 2005 por la Organización Mundial de la Salud, basándose en resultados de investigaciones clínicas, histopatológicas y moleculares ha decidido incluir al Queratoquiste odontogénico paraqueratinizado dentro del grupo de los tumores odontogénicos como Tumor Odontogénico Quístico Queratinizante (1).

Su formación es a partir del órgano del esmalte previamente a la maduración y calcificación de los tejidos dentales. Es una lesión de comportamiento clínico agresivo, con un alto potencial de crecimiento, comprometiendo gran parte de la estructura ósea maxilar antes de expresar algún signo clínico. Histopatológicamente este tumor exhibe un epitelio delgado paraqueratinizado, cuyas células de la capa basal se observan en empalizada, con todos sus núcleos al mismo nivel (2-5). Suelen diagnosticarse en el maxilar inferior en una proporción que va de 2 1 a 3-1 con respecto al maxilar superior. Suele encontrarse en mayor proporción en el sexo masculino que en el femenino. La mayor incidencia, según grupos de edad, se ubica entre la segunda y tercera década de vida (1-16).

Radiográficamente, esta lesión se presenta como una imagen radiolúcida, bien circunscrita que presenta bordes radiopacos delgados. Es posible observar multilocularidad, en especial en lesiones grandes, sin embargo la mayoría de las lesiones suelen ser uniloculares.

Esta lesión puede formar parte de un conjunto de anomalías conocido bajo el nombre de Síndrome de Gorlin-Goltz, en el que pueden presentar diferentes anomalías de origen cutáneas como los carcinomas basocelulares, quistes, tumores benignos, fosetas palmares, costilla bífida, y dentales como el prognatismo moderado, oftálmicas como el hipertelorismo, neurológicas como el retardo mental leve, calcificación de la hoz del cerebro, hidrocéfalo congénito, meduloblastomas en mayor proporción a lo normal y sexuales como hipogonadismo en el sexo masculino y quistes ováricos. El tratamiento es la enucleación quirúrgica, ante la posibilidad de poder recidivar y su diagnóstico diferencial es entre otros, el ameloblastoma, el fibroma ameloblástico y el quiste dentígero (7-9).

El objetivo de este trabajo es presentar un caso clínico de un Tumor Odontogénico Queratoquístico estudiado a través del diagnóstico clínico, radiográfico e histopatológico y su resolución quirúrgica.

\section{PRESENTACIÓN DEL CASO}

Paciente femenina de 26 años de raza caucásica, sin antecedentes de interés derivado por su odontólogo por una presunta movilidad dentaria del primer y segundo molar inferior derecho. El paciente relata que le habían realizado un tratamiento endodóntico 6 meses atrás en la pieza 4.6. El examen clínico reveló una leve movilidad en las piezas 4.6 y 4.7. La mucosa de revestimiento de la zona presentaba características de normalidad. (Fig. 1).

Se decide realizar una radiografía retroalveolar en la que se observa una radiolucidez asociada a las piezas 4.7 y 4.6 , éste con un tratamiento endodóntico y la pieza 4.8 con retención mucosa (Fig. 2).

A partir de lo expuesto se realizan un par radiográfico con la técnica panorámica en la que se observa

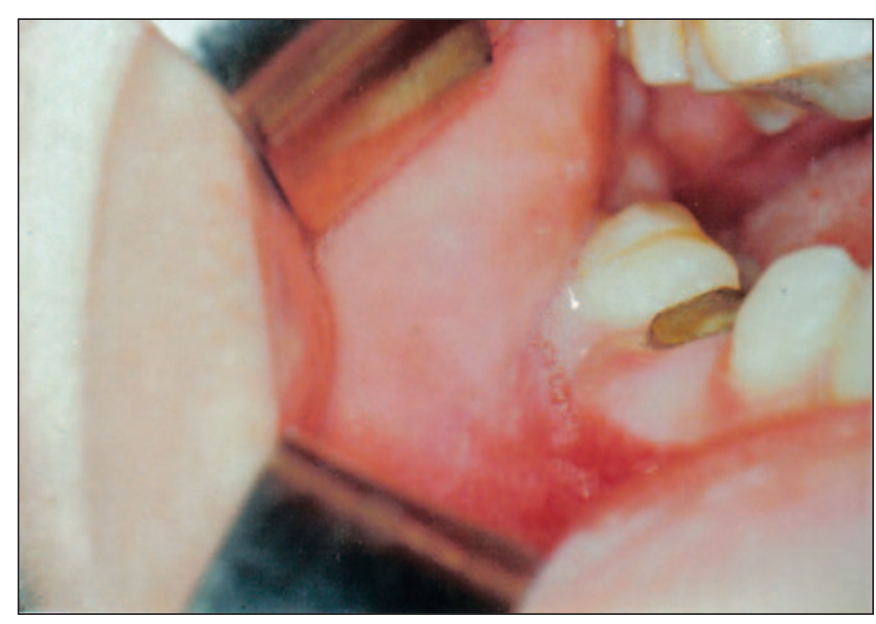

Fig. 1. Se observa la mucosa de revestimiento con características de normalidad. 


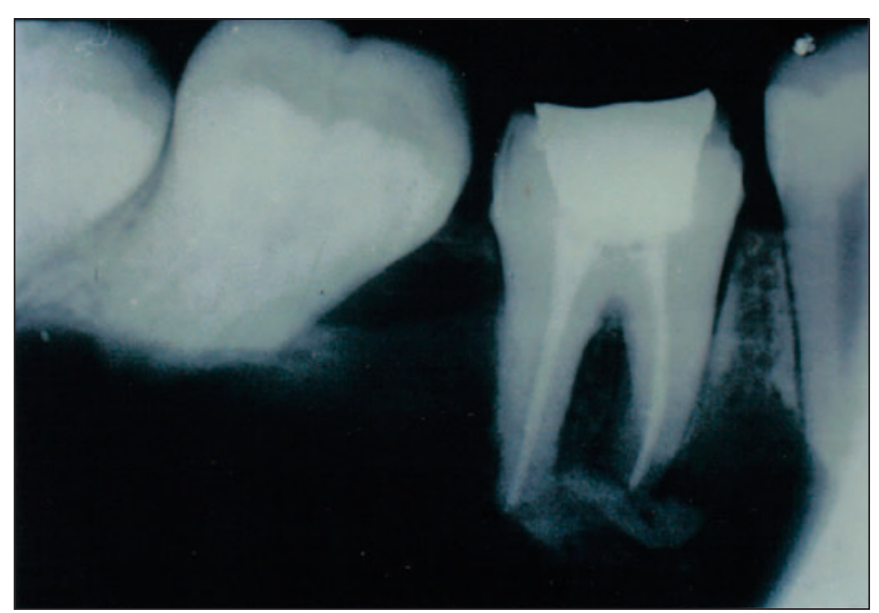

Fig. 2. Radiografía retroalveolar: se observa una zona radiolúcida que involucra al primer, segundo molar y parte del tercer molar inferior derecho.

en el sector posteroinferior derecho una zona radiolúcida unilocular que incluye la piezas $4.5,4.6,4.7$ y 4.8 en retención, el conducto dentario inferior se lo visualiza desplazado en cercanía a la basal mandibular (Fig. 3). Dadas las características de la lesión se procede a tomar una radiografía oclusal en el maxilar inferior, en un plano lateralizado con una incidencia de $90^{\circ}$ en la que se manifiesta un leve soplamiento de la tabla lingual en el sector posterior derecho (Fig. 4).

Una vez concluidos los estudios clínicos y radiográficos, se determinó como conducta realizar la biopsia para poder evidenciar la posible patología. Se realiza la exéresis de material con una trefina, observando en la cavidad remanente un contenido semisólido de color blanquecino (fig. 5).

La muestra fue enviada a la Cátedra de Anatomía patológica de la Facultad de Odontología de la Universidad de Buenos Aires. Microscópicamente se observó un fragmento de tejido duro, cilíndrico color blanco nacarado y múltiples fragmentos de tejido blando y superficie irregular, de color blanquecino con áreas ocres que presenta un tamaño de $1,7 \mathrm{x}$ $1,3 \times 0,3 \mathrm{~cm}$. El diagnóstico fue el de tumor odontogénico queratoquístico.

Previo a la segunda cirugía se colocan en el paciente los arcos de Erich en forma preventiva frente a una posible fractura durante y posterior al acto quirúrgico.

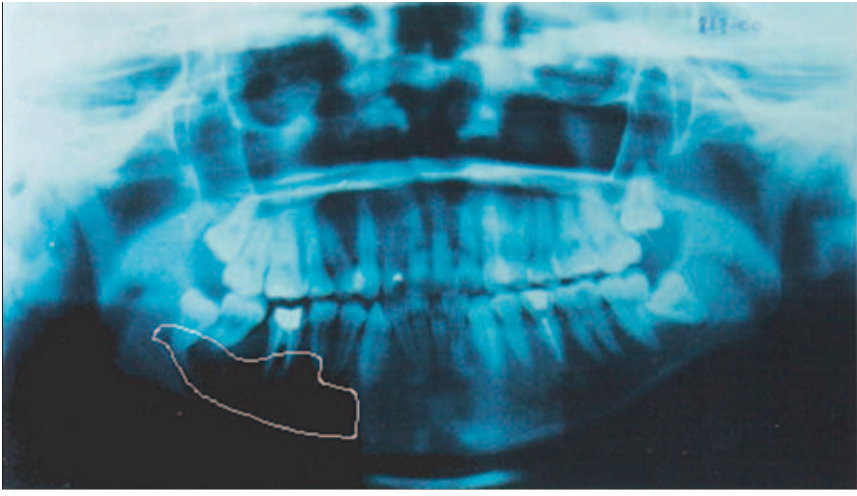

Fig. 3. Se observa contorneada una zona radiolúcida que abarca desde el tercer molar en retención hasta el segundo premolar inferior derecho.

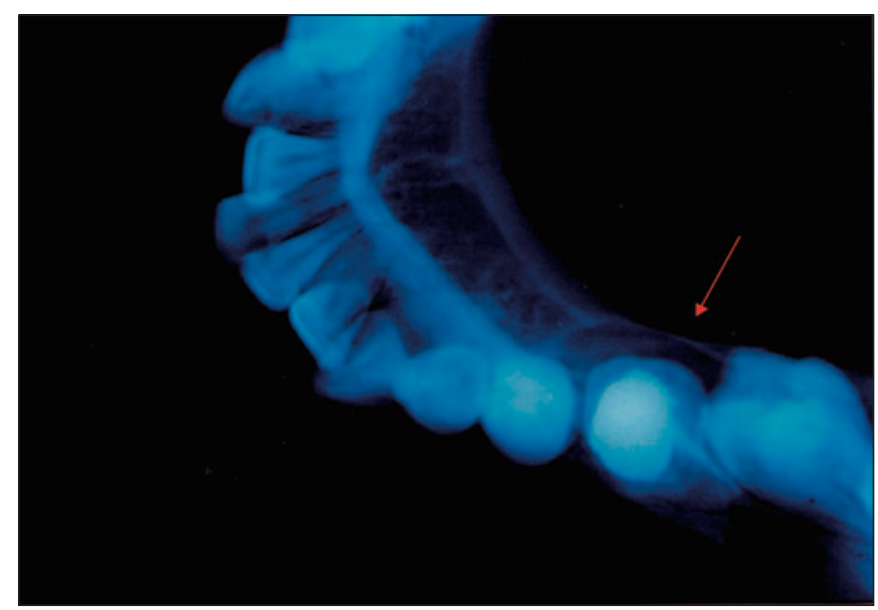

Fig. 4. Se observa leve soplamiento de la tabla lingual en el sector posterior derecho.

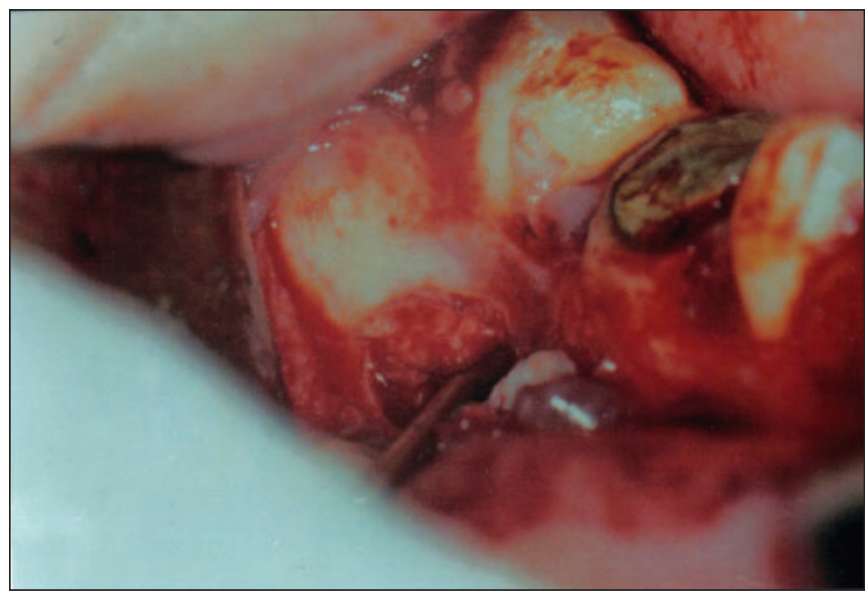

Fig. 5. Cavidad remanente posterior a al toma de la muestra realizada con una trefina. 
En el segundo procedimiento quirúrgico realizado con anestesia local, se abordó nuevamente la lesión realizando la exodoncia del primer premolar, los tres molares inferiores y la enucleación quística seguida de un tratamiento óseo afectado con la solución de Carnoy. (Figs. 6 y 7).

Se utilizó un hisopo de gasa embebida en la solución y se sujetó con una pinza mosquito durante 5 minutos, esta maniobra se repitió varias veces. El coágulo sanguíneo y el tejido necrótico que cubrió el defecto óseo fueron removidos con una cureta de hueso y se irrigó con solución fisiológica. Posterior a ello se realizó la sutura para lograr el cierre primario.

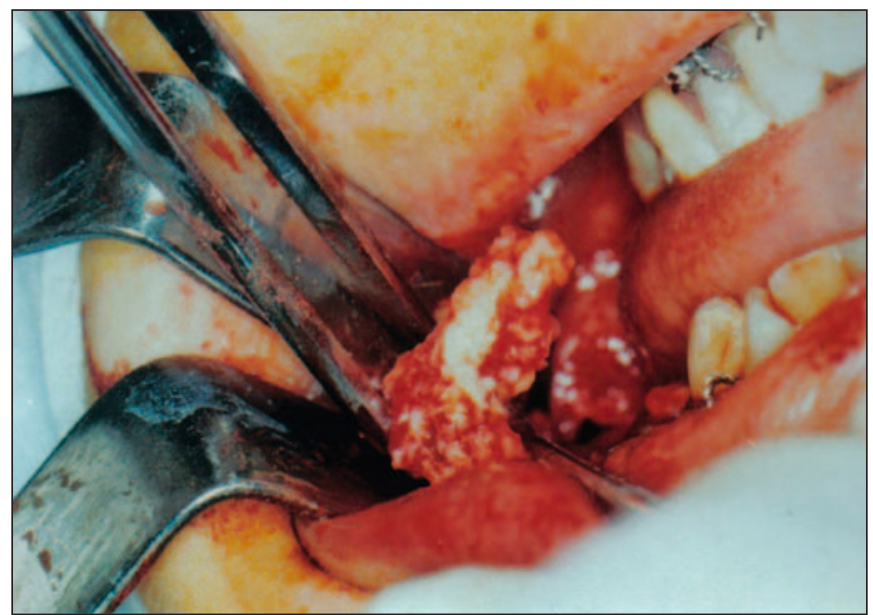

Fig. 6. Se observa la eliminación de la lesión.

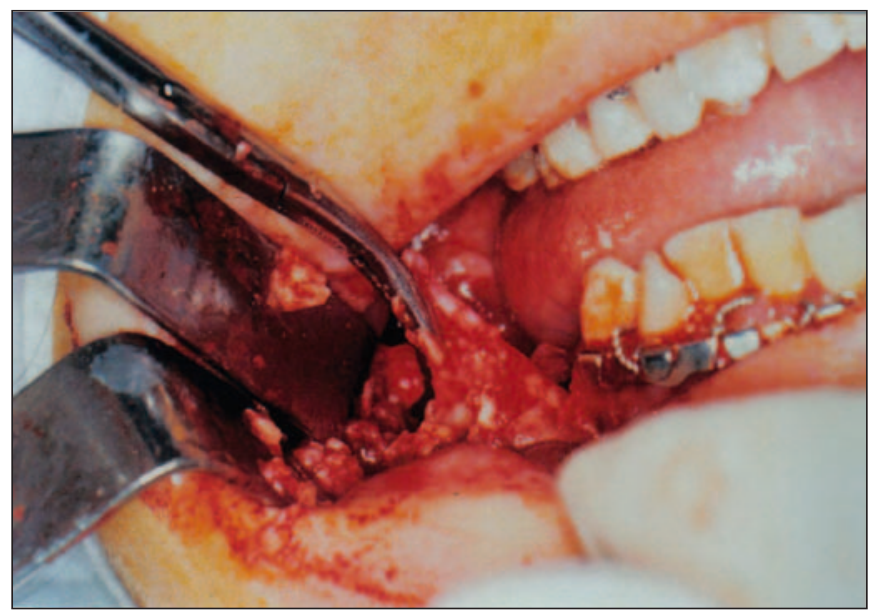

Fig. 7. En esta imagen se observa la membrana que cubría al tumor. Se visualizan los arcos de Erich colocados preventivamente en el caso de una fractura.
Fue enviado el material extraído para realizar los estudios histopatológicos.

Microscópicamente se observó fragmentos de cortical ósea, tejido óseo reaccional, medula fibrosa y tejido de granulación con abundante infiltrado inflamatorio crónico, zonas de epitelio hiperplásico e intenso infiltrado inflamatorio crónico. El diagnóstico definitivo fue el de tumor odontogénico queratoquístico.

Al paciente se le realizó controles periódicos a corto, mediano y largo plazo y no se constató ninguna recidiva de la lesión (Figs. 8 y 9).

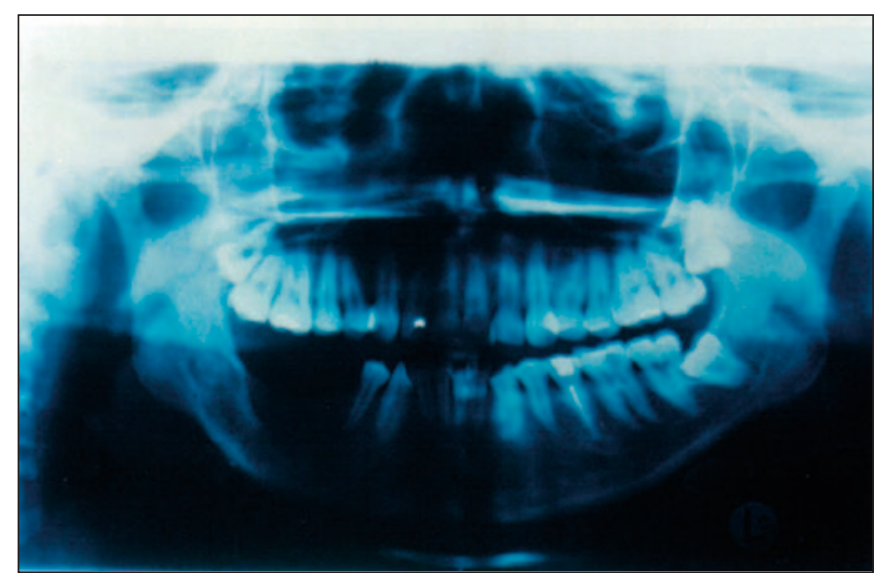

Fig. 8. Radiografía panorámica de control realizada al año, en la que se observa neoformación ósea en la zona en que se alojaba la lesión.

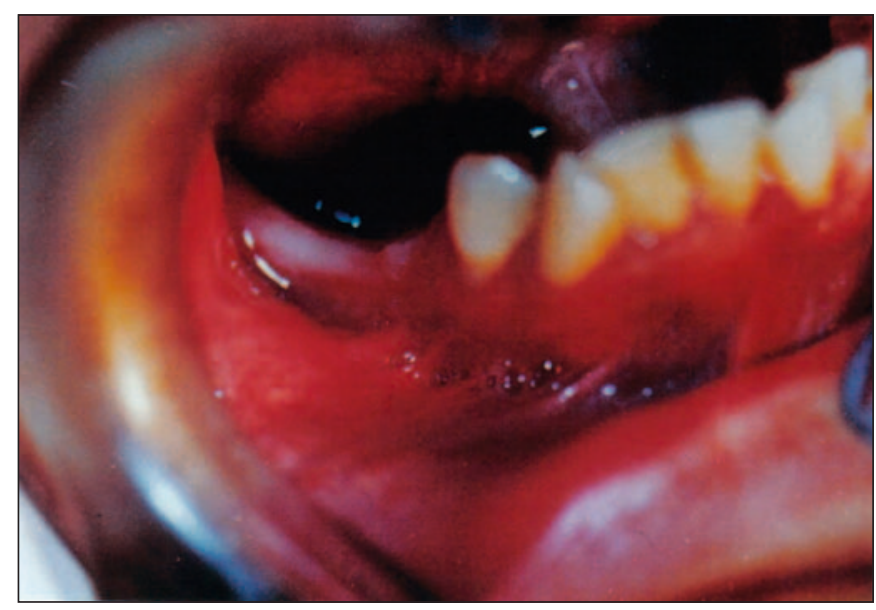

Fig. 9. Imagen clínica post operatoria a los 18 meses. Tejidos blandos reparados. 


\section{DISCUSIÓN Y CONCLUSIÓN}

Se debe destacar la importancia de un diagnóstico temprano de esta patología debido a la posibilidad de poder originar expansión e infiltración del hueso (7), asimetría facial, desplazamiento de los dientes y reabsorción radicular de las piezas adyacentes. La posibilidad de una radiografía extraoral como la técnica panorámica en el que nos brinda una visión completa de ambas arcadas dentarias y sus estructuras vecinas (13).

En el caso clínico presentado, el tumor odontogénico queratoquístico que se alojó en el maxilar inferior coincide estadísticamente en su ubicación con la bibliografía consultada. El paciente es del sexo femenino y tiene 26 años de edad, no coincidiendo con la literatura dado que hay una mayor predisposición en los varones y si coincidiendo con la edad del mismo ya que se suele encontrar esta lesión en personas que van desde la segunda hasta la tercera década de vida (1-12).

Por lo expuesto, se presentó un caso clínico con un tumor odontogénico queratoquístico y se han descrito las técnicas radiográficas útiles para su diagnóstico, como lo es la técnica intrabucal del paralelismo o de Fitzgerald por su nitidez e isometría, la oclusal con una incidencia a $90^{\circ}$ en el que nos permitió diagnosticar la expansión o rechazo de la tabla lingual y una radiografía extrabucal como la técnica panorámica que permitió visualizar la extensión y el compromiso óseo de la lesión. También es de destacar una correcta utilización de las normas de bioseguridad en el tratamiento quirúrgico, la prevención de una posible fractura intraoperatoria, colocando los arcos de Erich, dada la extensión del tumor y el cumplimiento de todos los pasos operatorios previamente planeados a la cirugía, la corroboración posterior a ella del diagnóstico presuntivo a través de los estudios anatomopatológicos y los seguimientos a corto, mediano y largo plazo de dicho paciente.

\section{BIBLIOGRAFÍA}

1. Sapp JP, Eversole LR, Wysocki GP. Patología Oral y Maxilofacial Contemporánea. Madrid España, Harcourt S.A., 2005;38.
2. Wrigtht JM. The odontogenic keratocyst: orthokeratinized variant. Oral Surg Oral Med Oral Pathol.1981;51(6):609-18.

3. Li TJ, Kitano M, Chen XM, Itoh T, Kawashima K, Sugihara K, Nozoe E, Mimura T. Orthokeratinized odontogenic cyst: a clinicopathological and immunocytochemical study of 15 cases. Histopathology. 1998; 32(3):242-51.

4. Kimi K, Kumamoto H, Ooya K, Motegi K . Immunohistochemical analysis of cell-cycle- and apoptosis-related factors in lining epithelium of odontogenic keratocysts. J Oral Pathol Med. 2001; 30(7):434-42.

5. MyoungH, HongSP, HongSD, LeeJI, LimCY, ChoungPH, LeeJH, ChoiJY, SeoBM, Kim MJ. Odontogenic Keratocyst: Review of 256 cases for recurrence and clinicopathologic parameters. Oral Surg Oral Med Oral Pathol Oral Radiol Endod.2001;91(3):328-33.

6. Kim do K, Ahn SG, Kim J, Yoon JH. Comparative $\mathrm{Ki}-67$ expression and apoptosis in the odontogenic keratocyst associated with or without an impacted tooth in addition to unilocular and multilocular varieties. Yonsei Med J. 2003;44(5): 841-6.

7. Pérez González E, López Labady J, Villarroel Dorrego M. Quiste odontogénico ortoqueratinizado: Una nueva entidad derivada del queratoquiste odontogénico. Revisión de la literatura Acta odontol. venez v.44 n.3 Caracas dic. 2006.

8. Keszler, A., Piloni, M.J. Malignant transformation in odontogenic keratocyst. Case Med Oral. 2002; 7(5):331-5.

9. Makowski GJ, Mc Guff S, Van Sickels, JE: Squamous cel carcinoma in a maxillary Odontogenic keratocyst. J Oral Maxillofac Surg 2001;59:7680 .

10. Williams C, Pemble W. Squamous cell carcinoma ex-odontogenic keratocyst: A Review of cases from de Armed Forces Institute of Pathology. Oral Surg Oral Med Oral Patho. Aug. 2002;94 (2):546. 
11. Chaisuparat R, Coletti E, Ord R, Nikitakis N. Primary Intraosseous ondontogenic Carcinoma arising in an odontogenic cyst or the novo: a clinicopathologic correlation of five cases. Oral Surg Oral Med Oral Patho. 2004;98(2): 140.

12. Rodríguez J H; Carbajal E. Estudio radiológico y tratamiento quirúrgico de un queratoquiste. C.A.O. 200;53:16-20.

13. Carbajal E, Vazquez D. Diagnostico por imágenes. Principios en odontología; Buenos Aires Argentina. Editorial El Escriba; 2009.

14. Pogrel MA. The history of the odontogenic keratocyst. Oral Maxillofacial Surg Clin N Am 2003;15:311-5.
15. Da Silva MJ, De Sousa SO, Corrêa L, Carvalhosa AA, De Araujo VC. Immunohistochemical study of the orthokeratinized odontogenic cyst: A comparison with the odontogenic keratocyst. Oral Surg Oral Med Oral Pathol 2002;94(6):732-7.

16. Sciubba JJ, Fantasia JE, Kahn LB. Tumors and cysts of the jaws. Armed Forces Institute of Pathology. Washington, D.C. 2001;34-43.

\section{CORRESPONDENCIA}

J. Vázquez Diego

Av. Corrientes 2362 2do piso Depto "C". C.P. (1046). Ciudad Autónoma de Buenos Aires. Argentina.

E-mail: jv983@hotmail.com.ar 